\title{
Effects of Fruit Coatings, Fungicide, and Storage Temperature on Fruit Shelf-Life and Qualities of 'California' Papaya
}

\author{
DOI: $10.18196 /$ pt.2018.074.1-8
}

\author{
Soesiladi E. Widodo ${ }^{1 *}$, Zulferiyenni ${ }^{2}$, Suskandini R. Dirmawati ${ }^{3}$, Rachmansyah A. Wardhana ${ }^{4}$, \\ Fitria $^{1}$, Annisa Fitri ${ }^{1}$, Jeanette Fajryah ${ }^{1}$ \\ ${ }^{1}$ Department of Agronomy and Horticulture, Faculty of Agriculture, University of Lampung, Bandar Lampung, Indonesia 35145 \\ ${ }^{2}$ Department of Agricultural Product Technology, Faculty of Agriculture, University of Lampung, Bandar Lampung, Indonesia 35145 \\ ${ }^{3}$ Department of Plant Protection, Faculty of Agriculture, University of Lampung, Bandar Lampung, Indonesia 35145 \\ ${ }^{4}$ Great Giant Foods, Co. Ltd., Terbanggi Besar, Central Lampung, Indonesia \\ *Corresponding author, email: sestiwidodo@gmail.com
}

\begin{abstract}
'California' papaya is a newly released papaya cultivar in Indonesia that has a very short shelf-life. Increasing demands in both local and export markets warrant the need for a proper postharvest technology to prolong its shelf-life. This research was conducted to study the effects of fruit coatings (chitosan, KD-112, plastic wrapping), fungicide Prochloraz, and storage temperatures on its fruit shelf-life and quality. Three parallel experiments were conducted with treatments arranged in a completely randomized design of a $2 \times 2 \times 2$ factorial design. The first factor was chitosan (with and without 1.25\% chitosan), or KD-112 (with and without 14\% KD-112), or plastic wrapping (with and without one layer plastic wrapping), the second was Prochloraz (with and without $0.67 \mathrm{~mL} / \mathrm{L}$ Prochloraz), and the third was storage temperature (27-28 and $\left.16-18^{\circ} \mathrm{C}\right)$. While Prochloraz did not affect fruit variables, cooler temperature and coatings lengthened fruit shelf-life with the best effect shown by plastic wrapping which lengthened fruit shelf-life by 13 days, without affecting fruit qualities. Significant effects of coating and cooler temperature determined their combined effects, with the best effect achieved by applying the three factors that lengthened fruit shelf-life by 11-23 days longer.
\end{abstract}

Keywords: Coating, Papaya, Prochloraz, Storage, Shelf-life

\section{ABSTRAK}

Pepaya 'California' adalah kultivar pepaya yang baru dirilis di Indonesia yang memiliki umur simpan yang sangat singkat. Meningkatnya permintaan di pasar lokal dan ekspor menjamin perlunya teknologi pasca panen yang tepat untuk memperpanjang umur simpannya. Penelitian ini dilakukan untuk mempelajari efek pelapisan buah (chitosan, KD-112, pembungkus plastik), fungisida Prochloraz, dan suhu penyimpanan pada umur dan kualitas buah buahnya. Tiga percobaan paralel dilakukan dengan perawatan yang diatur dalam desain acak lengkap dari desain faktorial $2 \times 2 \times 2$. Faktor pertama adalah chitosan (dengan dan tanpa 1,25\% chitosan), atau KD- 12 (dengan dan tanpa 14\% KD-1 12), atau pembungkus plastik (dengan dan tanpa satu lapisan pembungkus plastik), yang kedua adalah Prochloraz (dengan dan tanpa 0,67 mL / L Prochloraz), dan yang ketiga adalah suhu penyimpanan (27-28 dan 16-18 C). Sementara Prochloraz tidak mempengaruhi variabel buah, suhu dingin dan pelapisan memperpanjang umur simpan buah dengan efek terbaik ditunjukkan oleh pembungkus plastik yang memperpanjang umur simpan buah selama 13 hari, tanpa mempengaruhi kualitas buah. Efek signifikan dari pelapisan dan suhu dingin menentukan efek gabungannya, dengan efek terbaik yang dicapai dengan menerapkan tiga faktor yang memperpanjang umur simpan buah dengan 11-23 hari lebih lama.

Kata Kunci: Pelapisan buah, Pepaya, Prochloraz, Penyimpanan, Umur simpan

\section{INTRODUCTION}

'California' papaya is a newly released papaya cultivar in Indonesia (Farisi, 2011) that has a very short shelf-life with a quick decrease of fruit qualities due to high respiration and transpiration rates. Increasing demands in both local and export markets warrant the need for a proper postharvest technology to prolong its shelf-life and maintain its high fruit qualities.
Chitosan is increasingly known as a potent fruit surface-coating having both biodegradable and biofungicidal functions (Raqeeb et al., 2009; Yanti et al., 2009). It has been reported to be suitable for coating many fruits (Hernandez-Munoz et al., 2006; Widodo and Zulferiyenni, 2008; Widodo et al., 2010; Chutichudet and Chutichudet, 2011; Widodo et al., 2013; Widodo et al., 2015; Sun et 
al., 2010). Its effects in lengthening fruit shelf-life were due to less transpiration rate (Chutichudet and Chutichudet, 2011) and decreased respiration (Hernandez-Munoz et al., 2008). Widodo et al., (2016) confirmed that $1.25 \%$ chitosan was proven to be a potent fruit coating.

KD-112 is a sugar ester blend solution that is introduced as a fruit coating to delay pineapple ripening during its postharvest handlings (Sari et al., 2015). As with other sucrose polyester coatings, it is mainly used as biosurfactant (Neta et al., 2012) and its main effects are to decrease respiration and transpiration rates, ethylene production, and to delay fruit color development and softening (Sumnu and Bayindirli, 1997). During coating with sugar ester blend solution, due to low respiration and transpiration rates, fruits are expected to respond by having low fruit weight loss and softening rates, that was also reported by another coating (Zulferiyenni et al., 2015).

Amongs fruit coating practices, plastic wrapping is known as a common practice in postharvest handling of horticultural products due to its simplicity, effectivity, and economical reasons. It works by developing a modified athmospheric condition of low $\mathrm{O} 2$ and high $\mathrm{CO} 2$ inside the coating and providing a physical barrier to water vapor which promotes low respiration and transpiration rates (Workneh et al., 2012; Nasution et al., 2012) due to its lower permeabilities to athmospheric gases and water vapour (Nasution et al., 2012).

When combining chitosan or sugar ester blend solution of KD-112 with plastic wrapping, a significantly longer shelf-life and maintained high fruit qualities of 'California' papaya are expected. That is because chitosan, KD-112, and plastic wrapping, as fruit coatings, plays similar functions by lowering respiration and transpiration rates. However, energy that is released by respiration as heat and water vapor that is released by transpiration may be traped inside coating. When fruit shelf-life is extended, these develop a condition favouring pathogen buildup inside coating. Hamdayanty et al. (2012) and Sharma (2015) reported pathogen buildup when fruit shelf-life was extended more than 6 and 12 days storage due to application of fruit coatings. Hewajulige and Wijeratnam (2010) also reported that as ripening progresses, lengthening fruit shelf-life might increase the risk of pathogen buildup.

Prochloraz (N - propyl - N - [2 - (2, 4, 6 - trichlorophenoxy) ethyl] - $1 \mathrm{H}$ - imidazole -1 - carboxamide) $\mathrm{C}_{15} \mathrm{H}_{16} \mathrm{Cl}_{3} \mathrm{~N}_{3} \mathrm{O}_{2}$ is known as a broad spectrum fungicide (Vinggaard et al., 2006). It is one of the most important imidazole fungicides that was reported to degrade much slower at $\mathrm{pH} 7.0$ compared to pH 4.0 and 9.2 (Aktar et al., 2008). Since its development in the late 1970s, it retains its uses as a popular fungicide in many agroindustries because of its effectiveness in disease control. As a market demand to 'California' papaya fruit increases, its effectiveness in postharvest handling needs to be tested.

Application of lower temperature during postharvest handling does not only decrease metabolic processes, but also decreases development of fungi sporulation (Singh et al., 2012). Therefore, a longer shelf-life and maintained high fruit qualities of 'California' papaya are expected by combining fruit coating (chitosan or sugar ester blend solution of KD-112 or plastic wrapping) with fungicide Prochloraz and lower storage temperature. This research objectives were to study the effects of fruit coatings (chitosan, KD-112, plastic wrapping), fungicide Prochloraz, and storage temperatures on the fruit shelf-life and qualities of 'California' papaya.

\section{MATERIALS AND METHODS}

This research was conducted in July-September 2016 in the Horticultural Postharvest Laboratory, 
Faculty of Agriculture, University of Lampung, Bandar Lampung, Indonesia. 'California' papaya fruits at ripening stage I (green fruit with yellowing spot at peduncle side; Manenoi et al., 2007) were received as a fresh harvest directly from Nusantara Tropical Farm, Co. Ltd., Labuhan Ratu, East Lampung, Indonesia. Fruit samples were then sorted based on physical appearances (fruit weight and shapes) and maturity (fruit color). Other materials were chitosan (cosmetic grade), sugar ester blend of KD-112 (Kao, Co. Ltd.), plastic wrapping of LDPE $\left(\right.$ Best Fresh ${ }^{\circledR}$ ), Prochloraz, and other chemicals for chemical analyses.

Three parallel experiments were conducted with treatments arranged in a completely randomized design of a $2 \times 2 \times 2$ factorial design. The first factor was chitosan [without (CO) and with (C1) 1.25\% chitosan], or KD-112 [without (K0) and with (K1) $14 \% \mathrm{KD}-112$ ), or plastic wrapping [without (W0) and with (W1) one layer plastic wrapping], the second was Prochloraz [without (P0) and with (P1) $0.67 \mathrm{~mL} / \mathrm{L}$ Prochloraz), and the third was storage temperature [27-28 (T0) and $\left.16-18{ }^{\circ} \mathrm{C}(\mathrm{T} 1)\right]$. The experiments used five replications with one fruit each. The observation on fruit stage development was conducted daily, while the other observations were terminated once when the fruits reached stage IV (perfectly yellow/orange).

Chitosan was diluted in $0.5 \%$ acetic acid (Widodo et al., 2015; Zulferiyenni et al., 2015). KD-112 solutions were prepared by adding distilled water to KD-112 stock solution according to their applied concentrations. The fruits were quickly dipped in Prochloraz solution, air-dried, and then quickly dipped in chitosan or KD-112 solutions (or water in the control) or wrapped in one-layer of plastic wrapping. Treated fruits were then placed in a storage room of room temperature of $27-28^{\circ} \mathrm{C}$ or a cooler one of $16-18{ }^{\circ} \mathrm{C}$. The cool storage room temperature of $16-18{ }^{\circ} \mathrm{C}$ was the lowest possible temperature that could be achieved in the storage room of $5.8 \times 2.8 \times 3.15 \mathrm{~m}^{3}$ with four air conditioners, humidifiers, and a thermohygrometer.

Observations were made on fruit shelf-life, weight loss, firmness (with a penetrometer typed FHM-5, with a cylindrical point of $5 \mathrm{~mm}$ in diameter of Takemura Electric Work, Co. Ltd., Japan), soluble solid as oBrix (with an Atago N-1E hand refractometer), titratable acidity (titrated with 0.1 $\mathrm{N} \mathrm{NaOH}$ and phenolphthalein as an indicator, presented as g citric acid/100 g), and sweetness level (oBrix/acidity ratio). All data were analyzed with ANOVA, and then further tested with Least Significantly Difference (LSD) at 5\%.

\section{RESULTS AND DISCUSSION}

Without treatment of fruit coating, fungicide, and cooler storage, 'California' papaya fruits reached fruit ripening stage IV (perfectly yellow/ orange) at 5-7 days storage. As their ripening was progressed, the fruits were softened from 25.10 $\mathrm{kg} / \mathrm{cm}^{2}$ to $5.43-5.87 \mathrm{~kg} / \mathrm{cm}^{2}$, their soluble solid contents ( ${ }^{\circ}$ Brix) were increased from $9.20 \%$ to about $11 \%$, and without significant changes of acid content, their sweetness levels were increased significantly.

The results showed that while fungicide Prochloraz did not affect fruit variables, coatings and cooler temperature lengthened the shelf-life of 'California' papaya fruits (Table 1-3). 'California' papaya fruits show similar response to chitosan and sugar ester blend of KD-112, by which their shelf-life was extended by 3-4 days longer than the control (Table 1-2). The best effect shown by plastic wrapping which extended 'California' papaya fruit shelf-life by 13 days (Table 3), without affecting fruit qualities.

Fungicide application with $0.67 \mathrm{ml} / \mathrm{L}$ Prochloraz did not affect fruit shelf-life significantly (Table 1-3). It did not mean that the fungicide was 
not effective. Its ineffectiveness due to improper any sanitation prior to fungicide treatment, this concentration was unlikely, because Diczbalis et result indicated that the pathogen might exist and al. (2014) reported that $0.55 \mathrm{ml} / \mathrm{L}$ Prochloraz was need a longer incubation period to buildup. In effective for a disease control of papaya. In our re- fact, some samples were infected by anthracnose search, because the fruit samples were not received Colletrotichum gloeosporioides (Penz.) Sacc., but in

Table 1. Effects of Chitosan, Fungicide Prochloraz, and Storage Temperature on Fruit Shelf-Life and Qualities of 'California' Papaya

\begin{tabular}{|c|c|c|c|c|c|c|}
\hline Treatments & Shelf-life (days)* & Weight loss (\%)* & Firmness $\left(\mathrm{kg} / \mathrm{cm}^{2}\right)^{*}$ & ${ }^{\circ}$ Brix $(\%)^{*}$ & Acidity $(\mathrm{g} / 100 \mathrm{~g})^{*}$ & Sweetness* \\
\hline \multicolumn{7}{|l|}{ Chitosan (C): } \\
\hline Without (CO) & $11.20 b$ & $10.30 \mathrm{a}$ & $5.58 \mathrm{a}$ & $9.92 \mathrm{a}$ & $0.14 a$ & $70.42 \mathrm{a}$ \\
\hline $1.25 \%(\mathrm{C} 1)$ & $14.45 \mathrm{a}$ & $11.49 a$ & $6.78 \mathrm{a}$ & $10.11 \mathrm{a}$ & $0.14 a$ & $78.05 \mathrm{a}$ \\
\hline \multicolumn{7}{|l|}{ Prochloraz (F): } \\
\hline Control (F0) & $11.60 \mathrm{a}$ & $10.19 a$ & $5.36 b$ & $10.13 a$ & $0.14 \mathrm{a}$ & $73.17 \mathrm{a}$ \\
\hline $0.67 \mathrm{~mL} / \mathrm{L}(\mathrm{F} 1)$ & $14.05 \mathrm{a}$ & $11.60 \mathrm{a}$ & $7.00 \mathrm{a}$ & $9.90 \mathrm{a}$ & $0.14 a$ & $75.30 \mathrm{a}$ \\
\hline \multicolumn{7}{|l|}{ Temperature (T): } \\
\hline $28 \pm 1{ }^{\circ} \mathrm{C}(\mathrm{T} 0)$ & $8.05 b$ & $7.15 b$ & $5.10 \mathrm{~b}$ & $10.56 a$ & $0.14 a$ & $76.96 \mathrm{a}$ \\
\hline $16-18^{\circ} \mathrm{C}(\mathrm{T} 1)$ & $17.60 \mathrm{a}$ & $14.64 \mathrm{a}$ & $7.27 \mathrm{a}$ & $9.47 \mathrm{~b}$ & $0.14 a$ & $71.51 \mathrm{a}$ \\
\hline \multicolumn{7}{|l|}{ CxF: } \\
\hline COFO & $9.40 \mathrm{~b}$ & $9.13 \mathrm{a}$ & $5.32 \mathrm{~b}$ & $10.20 \mathrm{a}$ & $0.16 a$ & $65.41 \mathrm{~b}$ \\
\hline COF1 & $13.00 \mathrm{ab}$ & $11.48 \mathrm{a}$ & $5.85 b$ & $9.64 \mathrm{a}$ & $0.13 a$ & $75.44 \mathrm{a}$ \\
\hline C1F0 & $13.80 \mathrm{ab}$ & $11.26 \mathrm{a}$ & $5.39 \mathrm{~b}$ & $10.06 a$ & $0.13 a$ & $80.92 a b$ \\
\hline C1F1 & $15.10 \mathrm{a}$ & $11.72 \mathrm{a}$ & $8.16 \mathrm{a}$ & $10.15 a$ & $0.14 a$ & $75.17 \mathrm{ab}$ \\
\hline \multicolumn{7}{|l|}{$\mathrm{C} \times \mathrm{T}:$} \\
\hline СОТО & $6.70 \mathrm{~b}$ & $6.02 \mathrm{~b}$ & $6.13 b$ & $10.38 a b$ & $0.15 a$ & $72.12 \mathrm{a}$ \\
\hline СОТ1 & $15.70 \mathrm{a}$ & $14.60 \mathrm{a}$ & $5.04 \mathrm{~b}$ & $9.46 \mathrm{~b}$ & $0.14 a$ & $68.72 \mathrm{a}$ \\
\hline С1T0 & $9.40 \mathrm{~b}$ & $8.28 b$ & $8.40 \mathrm{a}$ & $10.74 \mathrm{a}$ & $0.14 a$ & 81.79 a \\
\hline C1T1 & $19.50 \mathrm{a}$ & $14.69 \mathrm{a}$ & $5.15 b$ & $9.47 \mathrm{~b}$ & $0.14 a$ & $74.30 \mathrm{a}$ \\
\hline \multicolumn{7}{|l|}{$\mathrm{F} \times \mathrm{T}:$} \\
\hline FOTO & $7.30 \mathrm{~b}$ & $6.83 \mathrm{~b}$ & $5.53 b$ & $10.65 \mathrm{a}$ & $0.14 a$ & 78.75 a \\
\hline F0T1 & $15.90 \mathrm{a}$ & $13.56 \mathrm{a}$ & $5.19 b$ & $9.61 \mathrm{ab}$ & $0.15 a$ & 67.58 a \\
\hline F1T0 & $8.80 \mathrm{~b}$ & $7.47 \mathrm{~b}$ & $9.01 \mathrm{a}$ & $10.47 \mathrm{ab}$ & $0.14 a$ & 75.17 a \\
\hline F1T1 & $19.30 \mathrm{a}$ & $15.73 \mathrm{a}$ & $5.00 \mathrm{~b}$ & $9.32 \mathrm{~b}$ & $0.13 a$ & 75.44 a \\
\hline \multicolumn{7}{|l|}{$\mathrm{C} \times \mathrm{F} \times \mathrm{T}:$} \\
\hline СОFОТО & $5.20 \mathrm{~d}$ & $5.02 \mathrm{~b}$ & $5.64 b$ & $10.78 \mathrm{a}$ & $0.16 a$ & $69.21 \mathrm{ab}$ \\
\hline C1F0T0 & $9.40 \mathrm{~cd}$ & $8.64 \mathrm{~b}$ & $5.42 b$ & $10.52 \mathrm{a}$ & $0.13 a$ & $88.28 \mathrm{a}$ \\
\hline COF1TO & $8.20 \mathrm{~cd}$ & $7.01 \mathrm{~b}$ & $6.62 b$ & $9.98 \mathrm{a}$ & $0.14 a$ & $75.03 \mathrm{ab}$ \\
\hline C1F1T0 & $9.40 \mathrm{~cd}$ & $7.93 \mathrm{~b}$ & $11.39 a$ & $10.96 \mathrm{a}$ & $0.15 a$ & $75.31 \mathrm{ab}$ \\
\hline COFOT1 & $13.60 \mathrm{bc}$ & $13.23 \mathrm{a}$ & $5.00 \mathrm{~b}$ & $9.62 \mathrm{a}$ & $0.16 a$ & $61.60 \mathrm{~b}$ \\
\hline C1F0T1 & $18.20 a b$ & $13.88 \mathrm{a}$ & $5.37 \mathrm{~b}$ & $9.60 \mathrm{a}$ & $0.14 a$ & $75.57 \mathrm{ab}$ \\
\hline COF1T1 & $17.80 a b$ & $15.95 \mathrm{a}$ & $5.07 \mathrm{~b}$ & $9.30 \mathrm{a}$ & $0.13 a$ & $75.85 a b$ \\
\hline C1F1T1 & $20.80 \mathrm{a}$ & $15.50 \mathrm{a}$ & $4.93 \mathrm{~b}$ & $9.34 \mathrm{a}$ & $0.14 a$ & $75.03 \mathrm{ab}$ \\
\hline
\end{tabular}

Remarks: *Values in the same column of each treatment followed by same letters were not significantly different at LSD 5\%. Values of fruit firmness, soluble solid content ('Brix), acidity, and sweetness ('Brix/acid ratio) at 0 day-storage was $25.10 \mathrm{~kg} / \mathrm{cm}^{2}, 9.20 \%, 0.15 \mathrm{~g} / 100 \mathrm{~g}$, and 63.12 , consecutively. 
general, the infection did not affect fruit shelf-life. fruit shelf-life might increase the risk of pathogen Hamdayanty et al. (2012) and Sharma (2015) re- buildup.

ported 6-12 days incubation periods for pathogen Lowering storage temperature to $16-18{ }^{\circ} \mathrm{C}$ exbuildup. Hewajulige and Wijeratnam (2010) also tended 'California' papaya fruit shelf-life by 8-10 reported that as ripening progresses, lengthening days longer than the control (Table 1-3). Lower tem-

Table 2. Effects of Sugar Ester Blend Of Kd-112, Fungicide Prochloraz, and Storage Temperature on Fruit Shelf-Life and Qualities of 'California' Papaya

\begin{tabular}{|c|c|c|c|c|c|c|}
\hline Treatments & Shelf-life (days)* & Weight loss (\%)* & Firmness $\left(\mathrm{kg} / \mathrm{cm}^{2}\right)^{*}$ & ${ }^{\circ}$ Brix $(\%)^{*}$ & Acidity $(\mathrm{g} / 100 \mathrm{~g})^{*}$ & Sweetness* \\
\hline \multicolumn{7}{|l|}{ KD-112 (K): } \\
\hline Without (KO) & $10.55 b$ & $8.56 b$ & $5.06 \mathrm{a}$ & $10.21 \mathrm{a}$ & $0.13 a$ & $84.88 \mathrm{a}$ \\
\hline $14 \%(\mathrm{~K} 1)$ & $14.30 \mathrm{a}$ & $12.04 \mathrm{a}$ & $4.76 a$ & $9.92 \mathrm{a}$ & $0.12 \mathrm{a}$ & $84.37 \mathrm{a}$ \\
\hline \multicolumn{7}{|l|}{ Prochloraz (F): } \\
\hline Control (F0) & $12.30 \mathrm{a}$ & $10.21 \mathrm{a}$ & $4.76 \mathrm{a}$ & $9.73 \mathrm{a}$ & $0.13 a$ & $79.87 \mathrm{a}$ \\
\hline 0.67 mL/L (F1) & $12.55 \mathrm{a}$ & $10.38 \mathrm{a}$ & $5.06 a$ & $10.41 \mathrm{a}$ & $0.12 \mathrm{a}$ & $89.38 \mathrm{a}$ \\
\hline \multicolumn{7}{|l|}{ Temperature (T): } \\
\hline $28 \pm 1{ }^{\circ} \mathrm{C}(\mathrm{T} 0)$ & $8.30 \mathrm{~b}$ & $6.86 \mathrm{~b}$ & $5.75 a$ & $9.75 a$ & $0.13 a$ & $76.76 b$ \\
\hline $16-18^{\circ} \mathrm{C}(\mathrm{T} 1)$ & $16.55 \mathrm{a}$ & $13.74 \mathrm{a}$ & $4.07 \mathrm{~b}$ & $10.38 \mathrm{a}$ & $0.12 \mathrm{a}$ & $92.49 \mathrm{a}$ \\
\hline \multicolumn{7}{|l|}{$\mathrm{K} \times \mathrm{F}:$} \\
\hline KOFO & $10.50 \mathrm{~b}$ & $8.70 \mathrm{~b}$ & $4.91 \mathrm{a}$ & $9.98 \mathrm{a}$ & $0.13 a$ & $79.44 \mathrm{a}$ \\
\hline K0F1 & $10.60 \mathrm{~b}$ & $8.42 \mathrm{~b}$ & $5.21 \mathrm{a}$ & $10.44 \mathrm{a}$ & $0.12 \mathrm{a}$ & $90.31 \mathrm{a}$ \\
\hline K1F0 & $14.10 a b$ & $11.73 \mathrm{a}$ & $4.61 \mathrm{a}$ & $9.47 a$ & $0.12 \mathrm{a}$ & $80.30 \mathrm{a}$ \\
\hline K1F1 & $14.50 \mathrm{a}$ & $12.35 \mathrm{a}$ & $4.91 \mathrm{a}$ & $10.37 \mathrm{a}$ & $0.12 \mathrm{a}$ & $88.44 \mathrm{a}$ \\
\hline \multicolumn{7}{|l|}{$\mathrm{K} \times \mathrm{T}:$} \\
\hline КОТО & $6.30 c$ & $5.09 \mathrm{~d}$ & $5.99 a$ & $9.72 \mathrm{a}$ & $0.14 \mathrm{a}$ & $73.03 \mathrm{~b}$ \\
\hline К0Т1 & $14.80 \mathrm{a}$ & $12.02 b$ & $4.14 b$ & $10.70 \mathrm{a}$ & $0.12 \mathrm{a}$ & $96.72 \mathrm{a}$ \\
\hline К1T0 & $10.30 \mathrm{~b}$ & $8.62 c$ & $5.52 \mathrm{a}$ & $9.78 \mathrm{a}$ & $0.13 a$ & $80.48 a b$ \\
\hline K1T1 & $18.30 \mathrm{a}$ & $15.46 \mathrm{a}$ & $4.00 \mathrm{~b}$ & $10.06 \mathrm{a}$ & $0.12 a$ & $88.26 a b$ \\
\hline \multicolumn{7}{|l|}{$\mathrm{F} \times \mathrm{T}:$} \\
\hline FOTO & $8.40 \mathrm{~b}$ & $6.95 b$ & $5.35 a b$ & $9.11 \mathrm{a}$ & $0.13 a$ & $70.30 \mathrm{~b}$ \\
\hline F0T1 & $16.20 \mathrm{a}$ & $13.47 \mathrm{a}$ & $4.17 b c$ & $10.34 \mathrm{a}$ & $0.12 \mathrm{a}$ & $89.43 a b$ \\
\hline F1T0 & $8.20 \mathrm{~b}$ & $6.76 \mathrm{~b}$ & $6.16 \mathrm{a}$ & $10.39 a$ & $0.13 a$ & $83.21 a b$ \\
\hline F1T1 & $16.90 \mathrm{a}$ & $14.01 \mathrm{a}$ & $3.97 c$ & $10.42 \mathrm{a}$ & $0.12 a$ & $95.55 \mathrm{a}$ \\
\hline \multicolumn{7}{|l|}{$\mathrm{K} \times \mathrm{F} \times \mathrm{T}:$} \\
\hline KOFOTO & $7.00 \mathrm{~d}$ & $5.54 \mathrm{de}$ & $5.87 \mathrm{ab}$ & 8.62 b & $0.13 a$ & $65.60 \mathrm{~b}$ \\
\hline K1F0T0 & $9.80 \mathrm{~cd}$ & 8.36 cde & $4.84 a b c$ & $9.60 \mathrm{ab}$ & $0.13 a$ & $75.01 \mathrm{ab}$ \\
\hline K0F1T0 & $5.60 d$ & $4.64 \mathrm{e}$ & $6.11 a$ & $10.82 a b$ & $0.14 a$ & $80.47 a b$ \\
\hline K1F1T0 & $10.80 \mathrm{bcd}$ & $8.87 \mathrm{~cd}$ & $6.20 \mathrm{a}$ & $9.96 \mathrm{ab}$ & $0.12 \mathrm{a}$ & $85.95 a b$ \\
\hline KOF0T1 & $14.00 \mathrm{abc}$ & $11.85 b c$ & $3.96 b c$ & 11.34 a & $0.12 a$ & $93.28 a b$ \\
\hline K1F0T1 & $18.40 \mathrm{a}$ & $15.09 \mathrm{ab}$ & $4.38 a b c$ & $9.34 a b$ & $0.11 \mathrm{a}$ & $85.59 a b$ \\
\hline K0F1T1 & $15.60 a b$ & $12.19 a b c$ & $4.32 a b c$ & $10.06 a b$ & $0.11 \mathrm{a}$ & $100.16 a$ \\
\hline K1F1T1 & $18.20 \mathrm{a}$ & $15.83 \mathrm{a}$ & $3.63 c$ & $10.78 a b$ & $0.12 a$ & $90.93 a b$ \\
\hline
\end{tabular}

Remarks: *Values in the same column of each treatment followed by same letters were not significantly different at LSD $5 \%$. Values of fruit firmness, soluble solid content ('Brix), acidity, and sweetness ('Brix/acid ratio) at 0 day-storage was $25.10 \mathrm{~kg} / \mathrm{cm}^{2}, 9.20 \%, 0.15 \mathrm{~g} / 100 \mathrm{~g}$, and 63.12 , consecutively. 
perature storage during postharvest handling might of fungi sporulation (Singh et al., 2012).

not only extended fruit shelf-life due to decreased Significant effects of coatings and cooler temmetabolic processes such as respiration and ethyl- perature determined their combined effects, with ene production (Workneh et al., 2012; Nasution et the best effect achieved by applying the three facal., 2012), but it might also decrease development tors that lengthened fruit shelf-life by 11-23 days

Table 3. Effects of Plastic Wrapping, Fungicide, and Storage Temperature on Fruit Shelf-Life and Qualities of 'California' Papaya

\begin{tabular}{|c|c|c|c|c|c|c|}
\hline Treatments & Shelf-life (days)* & Weight loss (\%)* & Firmness $\left(\mathrm{kg} / \mathrm{cm}^{2}\right)^{*}$ & ${ }^{\circ}$ Brix $(\%)^{*}$ & Acidity $(\mathrm{g} / 100 \mathrm{~g})^{*}$ & Sweetness* \\
\hline \multicolumn{7}{|l|}{ Wrapping (W): } \\
\hline Without (W0) & $10.25 b$ & $8.34 \mathrm{a}$ & $4.60 \mathrm{~b}$ & $10.49 a$ & $0.16 a$ & $68.96 \mathrm{a}$ \\
\hline One layer (W1) & $22.95 \mathrm{a}$ & $5.99 \mathrm{~b}$ & $10.60 \mathrm{a}$ & $10.60 \mathrm{a}$ & $0.14 a$ & $80.90 \mathrm{a}$ \\
\hline \multicolumn{7}{|l|}{ Prochloraz (F): } \\
\hline Control (F0) & $15.65 \mathrm{a}$ & $6.59 a$ & $6.98 \mathrm{a}$ & $10.70 \mathrm{a}$ & $0.15 a$ & $75.26 \mathrm{a}$ \\
\hline $0.67 \mathrm{~mL} / \mathrm{L}(\mathrm{F} 1)$ & $17.55 \mathrm{a}$ & $7.74 \mathrm{a}$ & $8.17 \mathrm{a}$ & $10.39 a$ & $0.15 a$ & $74.59 \mathrm{a}$ \\
\hline \multicolumn{7}{|l|}{ Temperature $(\mathrm{T})$ : } \\
\hline $28 \pm 1{ }^{\circ} \mathrm{C}(\mathrm{T} 0)$ & $12.45 \mathrm{~b}$ & $5.72 b$ & $8.41 \mathrm{a}$ & $10.60 \mathrm{a}$ & $0.15 a$ & $73.76 \mathrm{a}$ \\
\hline $16-18^{\circ} \mathrm{C}(\mathrm{T} 1)$ & $20.75 \mathrm{a}$ & $8.60 \mathrm{a}$ & $6.74 a$ & $10.49 a$ & $0.15 a$ & 76.09 a \\
\hline \multicolumn{7}{|l|}{ W x F: } \\
\hline WOFO & $9.50 \mathrm{~b}$ & $7.42 \mathrm{ab}$ & $4.54 \mathrm{~b}$ & $10.87 \mathrm{a}$ & $0.16 \mathrm{a}$ & $71.72 \mathrm{a}$ \\
\hline W0F1 & $11.00 \mathrm{~b}$ & $9.25 \mathrm{a}$ & $4.60 \mathrm{~b}$ & $10.11 \mathrm{a}$ & $0.16 a$ & $66.20 \mathrm{a}$ \\
\hline W1F0 & $21.80 \mathrm{a}$ & $5.76 b$ & $9.41 \mathrm{a}$ & $10.54 \mathrm{a}$ & $0.14 \mathrm{a}$ & $78.81 \mathrm{a}$ \\
\hline W1F1 & $24.10 \mathrm{a}$ & $6.23 b$ & $11.74 \mathrm{a}$ & $10.67 \mathrm{a}$ & $0.14 a$ & $82.98 \mathrm{a}$ \\
\hline \multicolumn{7}{|l|}{$\mathrm{W} \times \mathrm{T}:$} \\
\hline WOTO & $7.40 \mathrm{~d}$ & $6.16 b$ & $5.50 \mathrm{~b}$ & $10.11 \mathrm{a}$ & $0.16 a$ & $66.73 a$ \\
\hline W0T1 & $13.10 c$ & $10.51 \mathrm{a}$ & $3.70 \mathrm{~b}$ & $10.87 a$ & $0.16 a$ & $71.19 a$ \\
\hline W1T0 & $17.50 \mathrm{~b}$ & $5.29 \mathrm{~b}$ & $11.34 \mathrm{a}$ & $11.09 a$ & $0.15 a$ & $80.80 \mathrm{a}$ \\
\hline W1T1 & $28.40 \mathrm{a}$ & $6.70 \mathrm{~b}$ & $9.81 \mathrm{a}$ & $10.12 \mathrm{a}$ & $0.13 a$ & $81.00 \mathrm{a}$ \\
\hline \multicolumn{7}{|l|}{ Fx T: } \\
\hline FOTO & $11.70 \mathrm{~b}$ & $5.26 \mathrm{c}$ & $7.34 \mathrm{a}$ & $10.45 \mathrm{a}$ & $0.15 a$ & $75.75 a$ \\
\hline F0T1 & $19.60 \mathrm{a}$ & $7.93 \mathrm{ab}$ & $6.61 \mathrm{a}$ & $10.96 \mathrm{a}$ & $0.15 a$ & $74.78 \mathrm{a}$ \\
\hline F1T0 & $13.20 \mathrm{~b}$ & $6.19 b c$ & $9.50 \mathrm{a}$ & $10.75 \mathrm{a}$ & $0.16 a$ & $71.78 \mathrm{a}$ \\
\hline F1T1 & $21.90 \mathrm{a}$ & $9.29 a$ & $6.70 \mathrm{a}$ & $10.03 \mathrm{a}$ & $0.14 a$ & $77.41 \mathrm{a}$ \\
\hline \multicolumn{7}{|l|}{$\mathrm{W} \times \mathrm{F} \times \mathrm{T}:$} \\
\hline WOFOTO & $6.60 \mathrm{e}$ & $5.33 c$ & $5.43 b c$ & $10.48 a b$ & $0.16 a$ & $71.14 \mathrm{a}$ \\
\hline W1F0T0 & $16.80 \mathrm{bc}$ & $5.19 c$ & $9.30 \mathrm{ab}$ & $10.42 a b$ & $0.14 a$ & $80.36 \mathrm{a}$ \\
\hline WOF1TO & 8.20 de & $6.98 b c$ & $5.53 b c$ & $9.74 \mathrm{~b}$ & $0.17 a$ & $62.32 \mathrm{a}$ \\
\hline W1F1T0 & $18.20 \mathrm{~b}$ & $5.39 c$ & $13.42 \mathrm{a}$ & $11.76 \mathrm{a}$ & $0.15 a$ & $81.24 \mathrm{a}$ \\
\hline WOFOT1 & $12.40 \mathrm{~cd}$ & $9.53 \mathrm{ab}$ & $3.70 \mathrm{c}$ & $11.26 \mathrm{ab}$ & $0.16 a$ & $72.29 a$ \\
\hline W1F0T1 & $26.80 \mathrm{a}$ & $6.34 c$ & $9.60 \mathrm{ab}$ & $10.66 \mathrm{ab}$ & $0.14 a$ & $77.27 \mathrm{a}$ \\
\hline W0F1T1 & $13.80 \mathrm{bc}$ & $11.50 \mathrm{a}$ & $3.70 \mathrm{c}$ & $10.48 \mathrm{ab}$ & $0.16 a$ & $70.09 a$ \\
\hline W1F1T1 & $30.00 \mathrm{a}$ & $7.06 \mathrm{bc}$ & $10.06 \mathrm{ab}$ & $9.58 \mathrm{~b}$ & $0.12 \mathrm{a}$ & $84.73 \mathrm{a}$ \\
\hline
\end{tabular}

Remarks: *Values in the same column of each treatment followed by same letters were not significantly different at LSD $5 \%$. Values of fruit firmness, soluble solid content ('Brix), acidity, and sweetness ('Brix/acid ratio) at 0 day-storage was $25.10 \mathrm{~kg} / \mathrm{cm}^{2}, 9.20 \%, 0.15 \mathrm{~g} / 100 \mathrm{~g}$, and 63.12 , consecutively. 
longer (Table 1-3). The best effects was achieved by the combined application of fruit coating of one layer plastic wrapping, fungicide application of $0.67 \mathrm{~mL} / \mathrm{L}$ Prochloraz, and a cooler temperature storage of $16-18{ }^{\circ} \mathrm{C}$ that was capable of lengthening 'California' papaya fruit shelf-life up to 30 days storage, which was 23 days longer than the control.

When cooler temperature storage was not available, fruit coatings (chitosan or KD-112 or plastic wrapping) and fungicide Prochloraz could be applied during postharvest handling. The two combination were capable of lengthening 'California' papaya fruit by 4-8 days longer than the control (Table 1-3). Based on economical and simplicity considerations, however, a combination with one layer plastic wrapping was the best, because it was capable of extending fruit shelf-life of more than 8 days longer than the control (Table 3 ).

In general, the applied treatments did not affect fruit qualities, such as fruit weight loss, firmness, soluble solid and acid contents, and therefore, fruit sweetness (Table 1-3). Increased fruit weight loss due to lower temperature treatment might be an indirect effect of extended fruit shelf-life as also noted in our other results (Widodo and Zulferiyenni, 2008; Widodo, et al., 2013; Widodo et al., 2015; Widodo et al., 2016; Zulferiyenni et al., 2015). As noted in fruit shelf-life, significant effects of coatings and cooler temperature determined their combined effects in some variables, mostly in fruit weight loss and firmness.

\section{CONCLUSION}

While Prochloraz did not affect fruit variables, cooler temperature and coatings lengthened fruit shelf-life with the best effect shown by plastic wrapping which lengthened fruit shelf-life by 13 days, without affecting fruit qualities. Significant effects of coating and cooler temperature determined their combined effects, with the best effect achieved by applying the three factors that lengthened fruit shelf-life by 11-23 days longer. The best effects were achieved by the combined application of fruit coating of one layer plastic wrapping, fungicide application of $0.67 \mathrm{~mL} / \mathrm{L}$ Prochloraz, and a cooler temperature storage of $16-18{ }^{\circ} \mathrm{C}$ that was capable of lengthening 'California' papaya fruit shelf-life up to 30 days storage, which was 23 days longer than the control.

\section{ACKNOWLEDGEMENTS}

The authors would like to give special thanks to the General Directorate of Research Empower and Development, the Ministry of Research, Technology, and Higher Education, the Republic of Indonesia for funding this research through the National Research Grand of The Research for Science and Technology Development 2016. Thanks to Great Giant Foods, Co. Ltd., Terbanggi Besar, Central Lampung through Nusantara Tropical Farm, Co. Ltd., Labuhan Ratu, East Lampung, Indonesia for providing fruit samples and Prof. Dr. Muhammad Kamal, Drs. Dwi Hapsoro and Agus Karyanto for discussion during the preparations of research report and manuscript.

\section{REFERENCES}

Aktar, Md.W., D. Sengupta, S. Purkait, M. Ganguly, and M. Paramasivam. 2008. Degradation dynamics and dissipation kinetics of an imidazole fungicide (Prochloraz) in aqueous medium of varying pH. Interdisc. Toxicol. 1(3-4):203-205. Doi: 10.2478/v10102-010-0047-6.

Anonymous. 2010. Minister of Agriculture Decree Number: 2108/Kpts/ SR.120/5/2010, May 26, 2010, on the Release of Papaya Callina as a Superior Cultivar. The Minister of Agriculture, the Republic of Indonesia. 3pp.

Chutichudet, B., and P. Chutichudet. 201 1. Effects of chitosan coating to some postharvest characteristics of Hylocercus undatus (Haw) Brit. and rose fruit. Intl. J. Agric. Res. 6(1):82-92.

Diczbalis, Y., R. Henriod, D. Sole, and T. Campbell. 2014. Evaluation of the use of Prochloraz in the control of postharvest diseases of papaya in Australia. Acta Hort., 1022:133-142. Doi: 10.17660/ ActaHortic.2014.1022.17.

Farisi, K. A. 2011 . Strategy for Business Development of 'California' Papaya. Undergraduate Thesis, Department of Agribusiness, Faculty of Economics and Management, Bogor Agricultural Uni- 
versity, Bogor, Indonesia. 148pp. Https:// repository.ipb.ac.id/jspui/ bitstream/123456789/49945/1/H1 1 kaf.pdf. Downloaded: June 19,2018.

Hamdayanty, R. Yunita, N.N. Amin, and T.A. Damayanty. 2012. Pemanfaatan kitosan untuk mengendalikan antraknosa pada pepaya (Colletotrichum gloeosporioides) dan meningkatkan daya simpan buah [Use of chitosan to control antracnose on papaya (Colletotrichum gloeosporioides) and to extend its fruit shelf-life]. Jurnal Fitopatologi Indonesia 8(4):97-102.

Hernandez-Munoz, P., E. Almenar, V.D. Valle, D. Velez, and R. Gavara. 2008. Effect of chitosan combined with postharvest calcium treatment on strawberry (Fragaria x ananassa) quality during refrigerated storage. Food Chem. 110:428-435.

Hernandez-Munoz, P., E. Almenar, M.J. Ocio, and R. Gavara. 2006. Effect of calcium dips and chitosan coating on postharvest life of strawberries. Postharvest Biol. and Technol. 39:247-253.

Hewajulige, I.G.N., and S.W. Wijeratnam. 2010. Alternative postharvest treatments to control anthracnose disease in papaya during storage. Fresh Produce 1(1):15-20.

Manenoi, A., E.R.V. Bayongan, S. Thumdee, and R.E. Paull. 2007. Utility of 1-methylcyclopropane as a papaya postharvest treatment. Postharvest Biol. Technol. 44:55-62.

Nasution, I.S., Yusmanizar, and K. Melianda. 2012. Pengaruh penggunaan lapisan edibel (edible coating), kalsium klorida dan kemasan plastik terhadap mutu nanas (Ananas comosus Merr.) terolah minimal [Effects of edible coating, potassium chloride, and plastic wrapping on the qualities of minimally processed pineapple]. .). Teknol. dan Industri Pert. Indonesia 4(2): 1 -6.

Neta, N.A.S., J.C.S. Santos, S.O. Sancho, S. Radrigues, L.R.B. Goncalves, L.R. Rodrigues, and J.A. Teixeira. 2012. Enzymatic synthesis of sugar esters and their potential as surface-activestabilizers of coconut milk emulsions. J. Food Hydrocolloids 27:324- 331.

Raqeeb, A.A.E., T.M.M. Mahmud, S.R.S. Omar, A.R.M. Zaki, and A.R.A. Eryani. 2009. Effects of calsium and chitosan treatments on controlling anthracnose and postharvest quality of papaya (Carica papaya L.) Int. J. Agric. Res. 4(2):53-68.

Sari, R. M., S. E. Widodo, and S. Ratih. 2015. Pengaruh klorin dan pelapis buah pada tingkat kemasakan yang berbeda terhadap perkembangan stadium dan mempertahankan mutu buah nanas (Ananas comosus) kultivar MD2 [The effect of chlorine and fruit coatings applied at the different ripening levels on the fruit stadium development and maintaining qualities of pineapple fruit (Ananas comosus) cv. MD2] Paper presented at National Seminar on Science and Technology IV, University of Lampung, Bandar Lampung, Indonesia, $3^{\text {rd }}$ November 2015. Pp. 144-156.

Sharma, V. 2015. Evaluation of incidence and alternative management of post harvest fungal diseases of papaya fruits (Carica papaya L.) in Western U.P. Int. J. Theoretical \& App. Sci. 7(1):6-12.
Singh, P., A.K. Mishra, and N.N. Tripathi. 2012. Assessment of mycoflora associated with postharvest losses of papaya fruits. J. Agric. Technol. 8(3):961-968.

Sumnu, G., and L. Bayindirli. 1997. A review on preservation of fruits by sucrose polyester coatings," GIDA 22(3):227- 232.

Sun, D., G. Liang, J. Xie, X. Lei, and Y. Mo. 2010. Improved preservation effects of lichi fruit by combining chitosan coating with ascorbic acid treatment during postharvest storage. African J. Biotech. 9(22):3272-3279.

Vinggaard, A.M, U. Hass, M. Dalgaard, H.R. Andersen, E. Bonefeld-Jorgensen, S. Christiansen, P. Laier, and M.E. Poulsen. 2006. Prochloraz: an imidazole fungicide with multiple mechanisms of action. Int. J. Androl. 29(1):186-192.

Widodo, S.E., and Zulferiyenni. 2008. Aplikasi kitosan dalam teknologi pengemasan atmosfir termodifikasi buah duku [Chitosan applications in a modified atmosphere packaging technology of lanzone]. Paper presented at The National Seminar on Food 2008: Increasing Food Security Toward Global Market, Yogyakarta, Indonesia. Pp. 278-287.

Widodo, S.E., Zulferiyenni, and R. Arista. 2013. Coating effect of chitosan and plastic wrapping on the shelf-life and qualities of. 'Mutiara' and 'Crystal' guavas. J. ISSAAS 19(1):1-7.

Widodo, S.E., Zulferiyenni, Y.C. Ginting, F.H. Fazri, and D. Saputra. 2015. Effects of 1-methylcyclopropene and chitosan on the fruit shelf-life and qualities of two different ripening stages of 'Cavendish' banana. J. Food and Nutrition Sci. 3(1-2):54-59.

Widodo, S.E., Zulferiyenni, and D. Novaliana. 2010. Pengaruh chitosan terhadap mutu dan masa simpan pisang 'Muli' dan 'Cavendish' [Effects of chitosan on the fruit qualities and shelf-life of 'Muli' and 'Cavendish' bananas]. Paper presented at The 3rd National Seminar on Science and Technology, 18-19 October 2010, University of Lampung, Bandar Lampung, Indonesia. Pp. 503-512.

Widodo, S.E., Zulferiyenni, S.R. Dirmawati, R.A. Wardhana, Sunarti, and M.L. Wahyuni. 2016. Effects of chitosan and plastic wrapping on fruit shelf-life and qualities of 'California' papaya. Paper presented at The $6^{\text {th }}$ Annual Basic Science International Conference, Malang, Indonesia, June 7, 2016. Pp. 183-186.

Workneh, T.S., M. Azene, and S.Z. Tesfay. 2012. A review on the integrated agrotechnology of papaya fruit. African J. Biotech. 11 (85):1509815110.

Yanti, S.D., P.T. Nugroho, R. Aprisa, and E. Mulyana. 2009. The potential of chitosan as alternative biopesticide for postharvest plants. Asian J. Food Ag-Ind., Special Issue: S241-248.

Zulferiyenni, S.E. Widodo, and Y. Simatupang. 2015. Applications of 1 -methylcyclopropene and chitosan lengthened fruit shelf-life and maintained fruit qualities of 'Mutiara' guava fruits. J. Food and Nutrition Sci. 3(1-2):148-151. 